\title{
Comprehensive analysis of NAC transcription factors uncovers their roles during fiber development and stress response in cotton
}

\author{
Heng Sun, Meiling Hu, Jianying Li, Lin Chen, Meng Li, Shuqin Zhang, Xianlong Zhang and Xiyan Yang*
}

\begin{abstract}
Background: Transcription factors operate as important switches of transcription networks, and NAC (NAM, ATAF, and (UC) transcription factors are a plant-specific family involved in multiple biological processes. However, this gene family has not been systematically characterized in cotton.

Results: Here we identify a large number of genes with conservative NAC domains in four cotton species, with 147 found in Gossypium arboreum, 149 in G. raimondii, 267 in G. barbadense and 283 in G. hirsutum. Predicted membrane-bound NAC genes were also identified. Phylogenetic analysis showed that cotton NAC proteins clustered into seven subfamilies and homologous protein pairs showed similar characteristics. Evolutionary property analysis revealed that purifying selection of NAC genes occurred between diploid and polyploid cotton species, and variation analysis showed GhNAC genes may have been subjected to selection and domestication. NAC proteins showed extensive transactivation and this was dependent on the C-terminus. Some development and stress related cis-elements were enriched in the promoters of GhNAC genes. Comprehensive expression analysis indicated that 38 GhNAC genes were candidates for involvement in fiber development, and 120 in stress responses. Gene coexpression network analysis revealed relationships between fiber-associated NAC genes and secondary cell wall (SCW) biosynthesis genes.

Conclusions: NAC genes were identified in diploid and tetraploid cotton, revealing new insights into their evolution, variation and homology relationships. Transcriptome analysis and co-expression network indicated roles for GhNAC genes in cotton fiber development and stress response, and NAC genes may prove useful in molecular breeding programmes.
\end{abstract}

Keywords: Cotton, NAC, Evolutionary analysis, Transactivation, Fiber development, Stress response

\section{Background}

NAC (NAM, ATAF, and $\underline{\mathrm{CUC}}$ ) proteins are among the largest plant-specific transcription factor (TF) superfamily with 117 members in Arabidopsis, 151 in rice, 152 in soybean, and 251 in switchgrass [1-3]. In general, the structures of NAC proteins can be divided into two regions, the conserved $\mathrm{N}$-terminal DNA-binding domains (BD) and the high divergent $\mathrm{C}$-terminal transcriptional regulatory regions (TR) $[4,5]$. The $\mathrm{BD}$ contains about

* Correspondence: yxy@mail.hzau.edu.cn

National Key Laboratory of Crop Genetic Improvement, Huazhong

Agricultural University, Wuhan, Hubei 430070, People's Republic of China
150 160 amino acid residues and it can be further classified into five subdomains (A-E), associated with DNA binding, formation of homodimers or heterodimers and nuclear localization. The TRs are required to activate/repress transcription, and the high divergence amongst family members accounts for the diverse functions of NAC proteins [5]. Some NAC proteins have a transmembrane (TM) motif in the C-terminus, and show subcellular localization at the plasma membrane or endoplasmic reticulum; these proteins may be regulated by post-translational regulation under specific conditions [6-8]. Several species have membrane-bound NAC

(C) The Author(s). 2018 Open Access This article is distributed under the terms of the Creative Commons Attribution 4.0 International License (http://creativecommons.org/licenses/by/4.0/), which permits unrestricted use, distribution, and 
transcription factors, with 13 in Arabidopsis, 13 in tomato, 11 in soybean and 5 in rice, and most are induced by abiotic stresses [1, 9-12].

Previous studies showed that NAC proteins are widely implicated in the regulation of transcriptional reprogramming associated with diverse developmental processes [1316]. Mutation of $N A M$, the firstly identified $N A C$ gene, failed to develop a shoot apical meristem (SAM) in Petunia embryos [17]. Arabidopsis CUC1 and CUC2 showed a similar function in SAM formation [18]. In addition, NAC transcription factors play an important role in secondary cell wall (SCW) biosynthesis in plants $[13,19,20]$. Secondary wall biosynthesis-related NAC transcription factors, including VASCULAR-RELATED NAC-DOMAIN (VND) and NAC SECONDARY WALL THICKENING PROMOTING FACTOR1 (NST) are transcriptional switches that regulate SCW biosynthesis in Arabidopsis [13]. Seven $V N D$ genes $(V N D 1-7)$ were identified by an in vitro xylem vessel element inducible system, and microarray and promoter analysis revealed that these genes are up-regulated during in-vitro xylem vessel element formation and are specifically expressed in vascular cells [21]. Overexpressing $V N D$ genes up-regulated the expression of secondary wall-associated genes and showed thickened secondary walls [21, 22]. The NST genes (NST1-3) are preferentially expressed in xylary and extraxylary fibers, and regulate the formation of the SCW $[13,23]$. The function of secondary wall-related $N A C$ genes may be well conserved across the plant kingdom [13, 24, 25].

Plants are constantly confronted with a variety of abiotic stresses, such as drought and high salinity, and these stress conditions adversely affect plant growth, with a severe impact on agricultural productivity. To date, most evidence suggests that NAC proteins play roles in abiotic stress response. Many stress-responsive NAC proteins are positive regulators of abiotic stress tolerance, though some are negative regulators $[5,16,26]$. In Arabidopsis, AtRD26 (ANAC72) functions as a transcriptional activator in abscisic acid (ABA) mediated stress signaling [27]. Transgenic plants overexpressing ANAC019, ANAC055 or ANAC072 show enhanced drought tolerance by inducing several stress-inducible genes [28]. However, NAC016 negatively regulates drought tolerance by binding to the NAC016-specific binding motif (NAC16BM) in the AREB1 promoter and repressing the expression of AtAREB1. Meanwhile, NAP, encoded by a NAC016 target gene, binds to the promoter of AREB1 and suppresses its transcription [29]. Some stress-related NAC genes have been identified in rice and have potential for improving stress tolerance. Rice plants overexpressing STRESS-RESPONSIVE NAC1 (SNAC1) showed significantly improved drought and salt tolerance [30]. A stress-responsive NAC gene, SNAC3 positively regulates drought and heat tolerance in rice by modulating ROS homeostasis [31].
Cotton is one of the most important economic crops, and NAC proteins play important roles in cotton fiber development and stress tolerance. The process of fiber development can be divided into four continuous stages: initiation, elongation, secondary cell wall deposition and maturation [32]. The cotton NAC transcription factor, GhFSN1, was recently identified as a regulator of fiber SCW formation [25]. GhFSN1 is expressed in fibers during the SCW thickening stage, and transgenic experiments indicate that GhFSN1 positively regulates SCW thickness but negatively regulates fiber length. GhFSN1 directly regulates the expression of secondary cell wall-related genes in cotton. Other NAC genes have been identified as involved in stress tolerance in cotton. GhNAC79 with relatively higher transcript accumulation at later stages of cotyledon and fiber development, overexpression of GhNAC79 showed improved drought tolerance in both cotton and Arabidopsis [33]. A NAC transcription factor gene, GhATAF1 has been reported to play a role in crosstalk between biotic and abiotic stress responses [34]. Cotton plants overexpressing GhATAF1 show improved salt stress tolerance, but also an increased susceptibility to the fungal pathogens Verticillium dahliae and Botrytis cinerea. Previous genetic studies identified stress-responsive $N A C$ genes in cotton. GhNAC1 - GhNAC6 were identified by sequence homology, and these genes are differentially regulated under abiotic stress [35]. Seven GhNAC genes, GhNAC7 GhNAC13, which are preferentially expressed in roots, have been shown to be involved in stress responses [36]. The expression patterns of 60 GhNAC genes, which were located on D sub-genome, were analyzed during stresses and phytohormones [37]. We speculate that NAC proteins show functional conservation in SCW formation and stress response in plants. Although a few $N A C$ genes involved in fiber development and stress response have been characterized in cotton, the regulatory networks of $N A C$ genes are still poorly understood.

Genome-wide analysis of $N A C$ genes allows gene functional studies. The genome data for two diploid cotton species (G. arboreum, G. raimondii) and two tetraploid species (G. barbadense, G. hirsutum), and public transcriptome data sets have made possible the genome-wide identification and analysis of the gene families in cotton [38-42]. However, the NAC gene family is poorly characterized, especially in Upland cotton (G. hirsutum), which accounts for more than $90 \%$ of cultivated cotton worldwide; although such an analysis has been performed in diploid cotton [43, 44]. In the present study, the NAC gene family was comprehensively studied in four cotton species, to reveal phylogenetic relationships, synteny, genetic variation, co-expression networks, transactivation and cis-element analysis. We also linked $N A C$ gene family members to fiber development and stress responses. 


\section{Results}

Genome-wide identification of NAC family genes in Gossypium spp

Based on the PlantTFDB database and multiple sequence alignment analysis, we identified multiple non-redundant and complete $N A C$ genes in four cotton species, with 147 found in Gossypium arboreum, 149 in G. raimondii, 267 in G. barbadense and 283 in G. hirsutum (Additional file 1: Table S1). The predicted cotton NAC protein sequence lengths, molecular weights and protein isoelectric points (pI) were analyzed (Additional file 2: Figure S1, Additional file 3: Table S2). For example, the full length of GhNAC proteins range from 168 to 1219 amino acids, with molecular weights ranging from 20.1$135.6 \mathrm{kDa}$, and isoelectric points from 4.39 to 9.8. Multiple sequence alignment and conservative motif analysis showed that cotton NAC proteins possess a conserved NAC domain, which includes five subdomains (A-E), with some conserved amino acid present (Fig. 1a). A phylogenetic tree was constructed to gain insights into the evolutionary relationships between NAC proteins in Gossypium. NAC proteins from four cotton species group into seven subfamilies (I-VII), and each subfamily contains the NAC proteins derived from four cotton species (Fig. 1b).
There are 13, 12, 21 and 22 predicted membrane-bound NAC proteins were identified in four cotton species respectively (Red color in Additional file 1: Table S1). Most of the predicted membrane-bound NAC proteins had one TM at the C terminal, such as GhNAC157 (Additional file 4: Figure S2a). GaNAC111 possesses two predicted TM at the $\mathrm{C}$ terminal, and eight NAC proteins (GaNAC68, GaN AC57, GrNAC79, GbNAC23, GbNAC104, GbNAC184, GhNAC258, GhNAC169) have one TM preceding the conserved NAC domain (Additional file 4: Figure S2b, c). The predicted membrane-bound NAC proteins are mainly distributed in subfamilies II and III (46 out of 68), with none in subfamily VI. Phylogenetic analysis of these NAC proteins compared with the membrane-bound NAC proteins in Arabidopsis, tomato and rice show that some clade genes are present in diverse species (Additional file 4: Figure S2d).

Gene structure analysis based on exon/intron organization is an important and helpful method for studying genetic evolution. Most GhNAC genes had three exons $(193 / 283,68.2 \%)$ (Fig. 2a), though the homologous gene pair GhNAC111 and GhNAC253 contained only one exon, and GhNAC4 possessed the highest number with seventeen exons. GhNAC genes in the same subfamily showed conserved structural

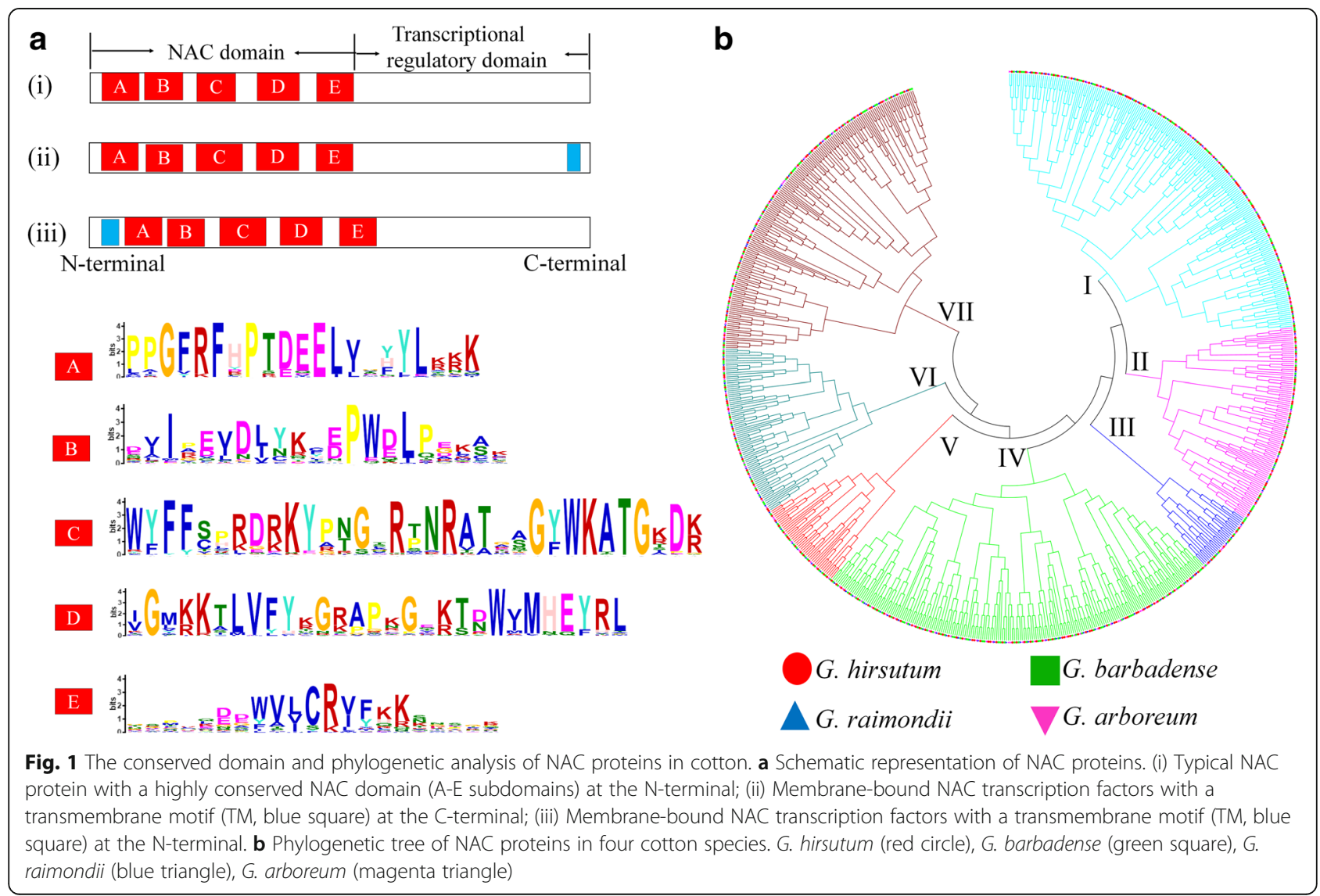



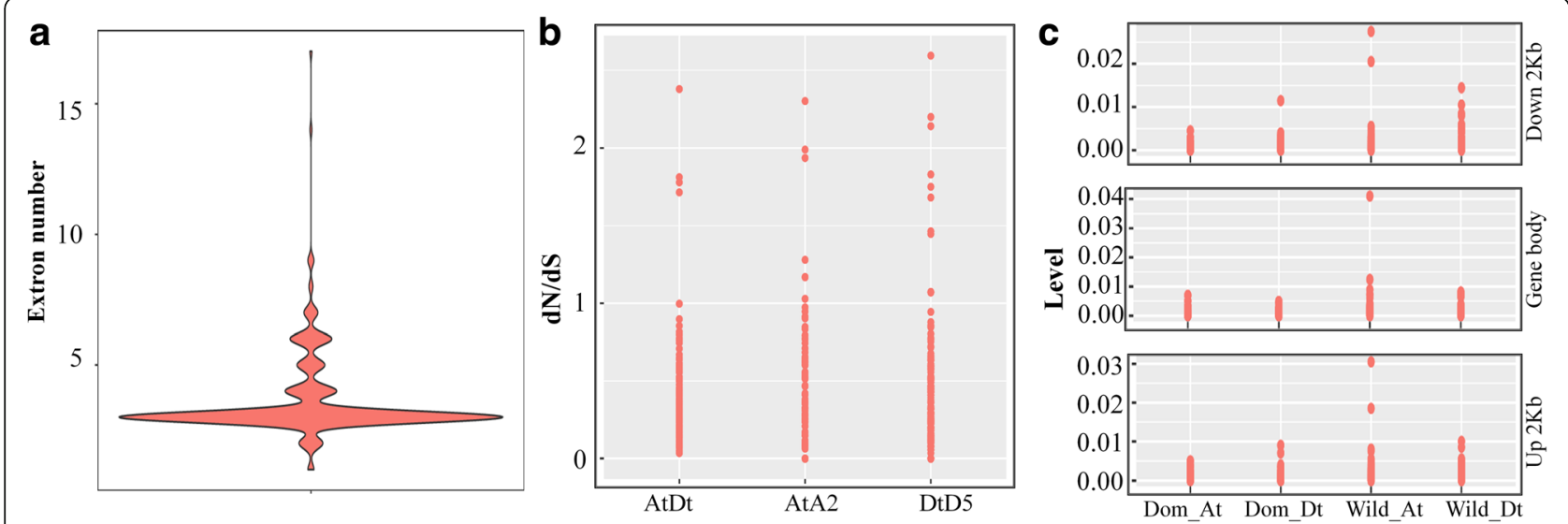

Fig. 2 Evolutionary analysis of NAC proteins in cotton. a Statistical analysis of exon numbers of NAC genes in G. hirsutum. b The ratio of nonsynonymous to synonymous substitutions $\left(\mathrm{d}_{N} / \mathrm{d}_{\mathrm{S}}\right.$ ) of NAC genes in inter-genomic (At Dt) and intra-genomic (A2 At and D5 Dt). c The SNP density of GhNAC genes in Upland cotton populations. The gene body, $2 \mathrm{~kb}$ upstream and $2 \mathrm{~kb}$ downstream sequences of each GhNAC gene were selected for analysis

similarities, and almost all of the most closely related gene pairs shared the same structural features.

\section{Genome synteny and variation analysis of NAC genes}

Genome synteny analysis is important for deciphering a genome's evolutionary history. We examined the distribution and gene homology of NAC genes by a genome synteny analysis (Additional file 5: Figure S3). For gene loci analysis, we found all chromosomes possessed at least one $N A C$ gene in At (A subgenome, with lower-case ' $t$ ' denoting tetraploid) and Dt. In G. hirsutum, more NAC genes are distributed on chromosomes A05, A11, D05 and D11 than on other chromosomes, while 25 GhNAC genes have no distinct chromosome location and could not be accurately mapped (Additional file 6: Table S3). We further examined homologous gene pairs by multiple homologous comparison analysis, and our results showed that there are 114, 138, 136 homologous gene pairs were identified from At to Dt, At to A2, Dt to D5, respectively (Additional file 7: Table S4). Furthermore, we found homologous genes in different cotton species show close evolutionary relationships and gene characteristics, such as gene structure and predicted protein molecular weight.

The non-synonymous $\left(\mathrm{d}_{\mathrm{N}}\right)$ and synonymous $\left(\mathrm{d}_{\mathrm{S}}\right)$ substitution rates within and between cotton species were calculated to explore evolutionary dynamics and selection pressures. Most of the $d_{N}$ is less than $d_{S}\left(d_{N} / d_{S}<1\right)$ in the inter-genomic (At and Dt) and intra-genomic (A2 and At or D5 and Dt) comparisons, suggesting that purifying selection of $N A C$ genes has occurred between both diploid and polyploid cotton species (Fig. 2b).

Upland cotton is the main cultivated cotton worldwide, and natural selection and domestication have played important roles in its development. Single nucleotide polymorphism (SNP) density in the gene body, and the regions $2 \mathrm{~kb}$ upstream and $2 \mathrm{~kb}$ downstream of GhNAC genes were analyzed based on the genome data for 31 wild cotton and 321 domesticated Upland cotton accessions. As expected, the SNP density of these three regions in wild cotton was higher than that of cultivated cotton, indicating that the evolution of cultivated cotton experienced selection and domestication. Moreover, the variant range of A subgenome was higher than that of the D subgenome in wild cotton, while the SNP density of $\mathrm{D}$ subgenome was higher than that of the A subgenome in the $2 \mathrm{~kb}$ upstream and $2 \mathrm{~kb}$ downstream regions in cultivated cotton (Fig. 2c).

\section{Cis-element analysis of NAC genes in G. hirsutum}

Promoter analysis is an effective method to study potential transcriptional regulation of genes. There are abundant regulatory elements existing in the promoter region of GhNAC genes, which are predicted to be involved in phytohormone responses, development and stress responses (Fig. 3, Additional file 8: Table S5). The 5 most commonly found cis-elements include the heat stress response element (HSE, 221 out of 283, 78.1\%), TC-rich repeats (76.7\%), G-box (69.6\%), GT1-motif (68.9\%), and circadian (68.6\%). HSE and TC-rich repeats are involved in defense and stress responsiveness, while circadian and GT1-motif are involved in light response and development. Our results implied that GhNAC genes might play an important role in both stress response and development in cotton.

\section{Identification of fiber-associated NAC family members in G. hirsutum}

The available and public transcriptome data sets have made it possible to comprehensively analyze NAC genes expressed during development processes in cotton. We 


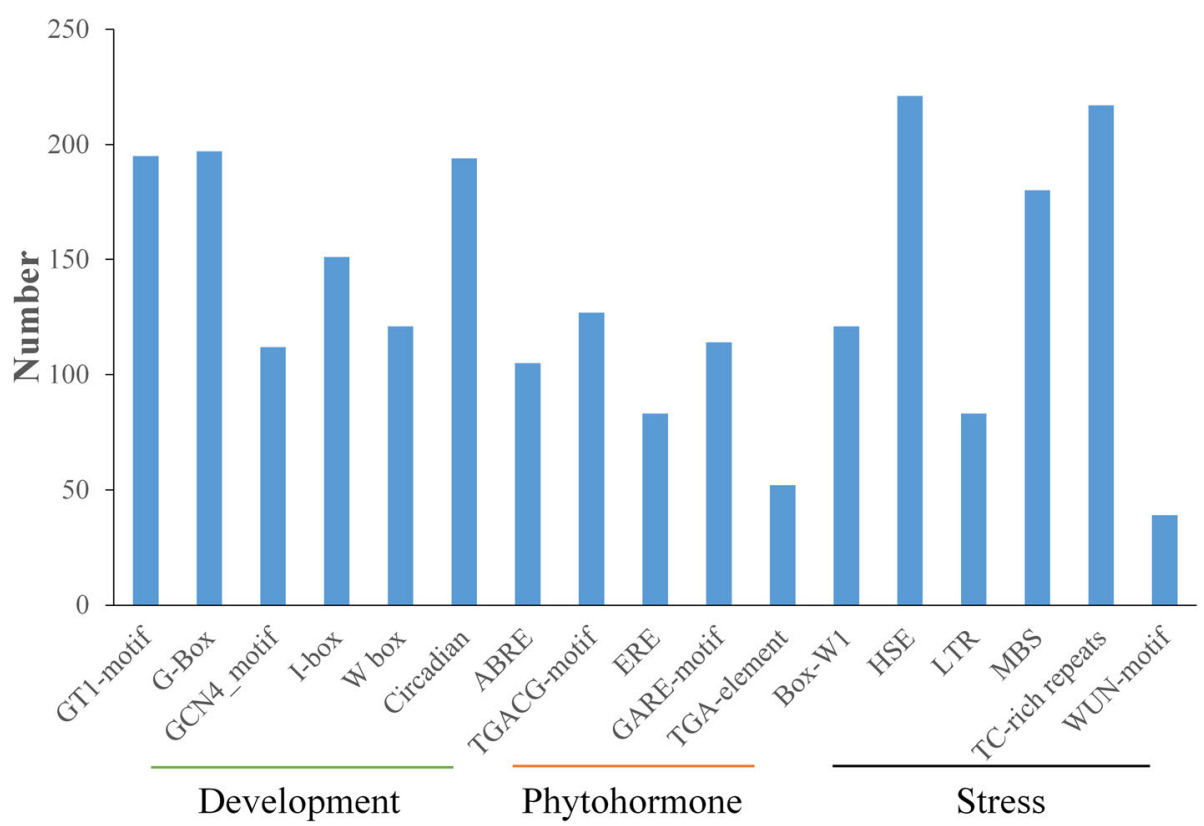

Fig. 3 Cis-element analysis of NAC genes in G. hirsutum. Cis-element analysis of GhNAC gene promoter. The 1.5-kb upstream sequence of the start codon of each NAC gene were analyzed by the PLANTCARE database

collected the transcriptome data sets from different tissues/organs, including the root, stem, leaf, petal, anther, stigma, ovule, seed and fiber (Additional file 9: Figure S4a) and identified 198 (70\%) GhNAC genes expressed at least in one tissue, and 37 (13.1\%) genes were expressed in all tissues (FPKM $\geq 1$ ). Some GhNAC genes were highly expressed (FPKM $\geq 20$ ) in stem, ovule and fiber (Fig. 4a).

Cotton fibers are the most important natural textile materials worldwide. The transcriptome data were analyzed during fiber development over four developmental stages (5 DPA, 10 DPA, 20 DPA and 25 DPA). There are $142(50.2 \%)$ GhNAC genes identified as being expressed during fiber development. The expression patterns of all GhNAC genes during fiber development were divided into four subclusters (cluster I-IV) (Fig. 4b). GhNAC genes belonging to subclusters I-III showed expression changes during fiber development. Genes from cluster I were highly expressed at 20 and 25 DPA, genes from cluster II showed low expression levels at early stages of development and then up-regulated at 25 DPA, while genes from cluster III were up-regulated at 20 DPA and then down-regulated at $25 \mathrm{DPA}$. Genes from cluster IV showed a high expression levels during 10 DPA and then low expression levels subsequently.

We identified 38 highly expressed (FPKM $\geq 20)$ GhNAC genes with obvious expression changes during fiber development, and these genes are evenly distributed in the A and D subgenomes (Fig. 4c, Additional file 10: Table S6). There are 13 homologous pairs within these genes, and two genes of each homologous gene pair showed similar expression trends. We found ten genes (GhNAC33, GhNAC39, GhNAC108, GhNAC125, GhNAC166, GhNA C193, GhNAC217, GhNAC251, GhNAC273, GhNAC280) that showed preferential expression in fibers compare with other tissues or organs. Two genes (GhNAC7, Gh NAC151) showed high expression at the elongation stage and SCW thickening stage (10-25 DPA), 12 genes (GhNAC33, GhNAC43, GhNAC74, GhNAC95, GhN AC106, GhNAC129, GhNAC188, GhNAC193, GhNAC217, GhNAC237, GhNAC249, GhNAC270) showed high expression at the transition and SCW thickening stage (2025 DPA), eight genes (GhNAC42, GhNAC47, GhNAC56, GhNAC120, GhNAC173, GhNAC178, GhNAC195, Gh $N A C 262)$ showed high expression specifically at the SCW thickening stage (25 DPA) and nine genes (GhNAC37, GhNAC108, GhNAC125, GhNAC133, GhNAC141, Gh NAC182, GhNAC251, GhNAC273, GhNAC280) showed specific high expression at the transition stage. Three genes (GhNAC125, GhNAC166, GhNAC273) have high homology with Arabidopsis NST1, which may regulate the formation of SCW [20].

Previous reports showed some genes in the At and Dt subgenomes demonstrated biased expression patterns. Of the 38 genes, we found the expression of some GhNAC homoeologous genes demonstrated biased expression patterns. GhNAC39 and GhNAC125 located in the At subgenome, have preferential expression in fibers, whereas the expression levels of the Dt homoeologous genes were nearly undetectable (Fig. 4d). We investigated the 


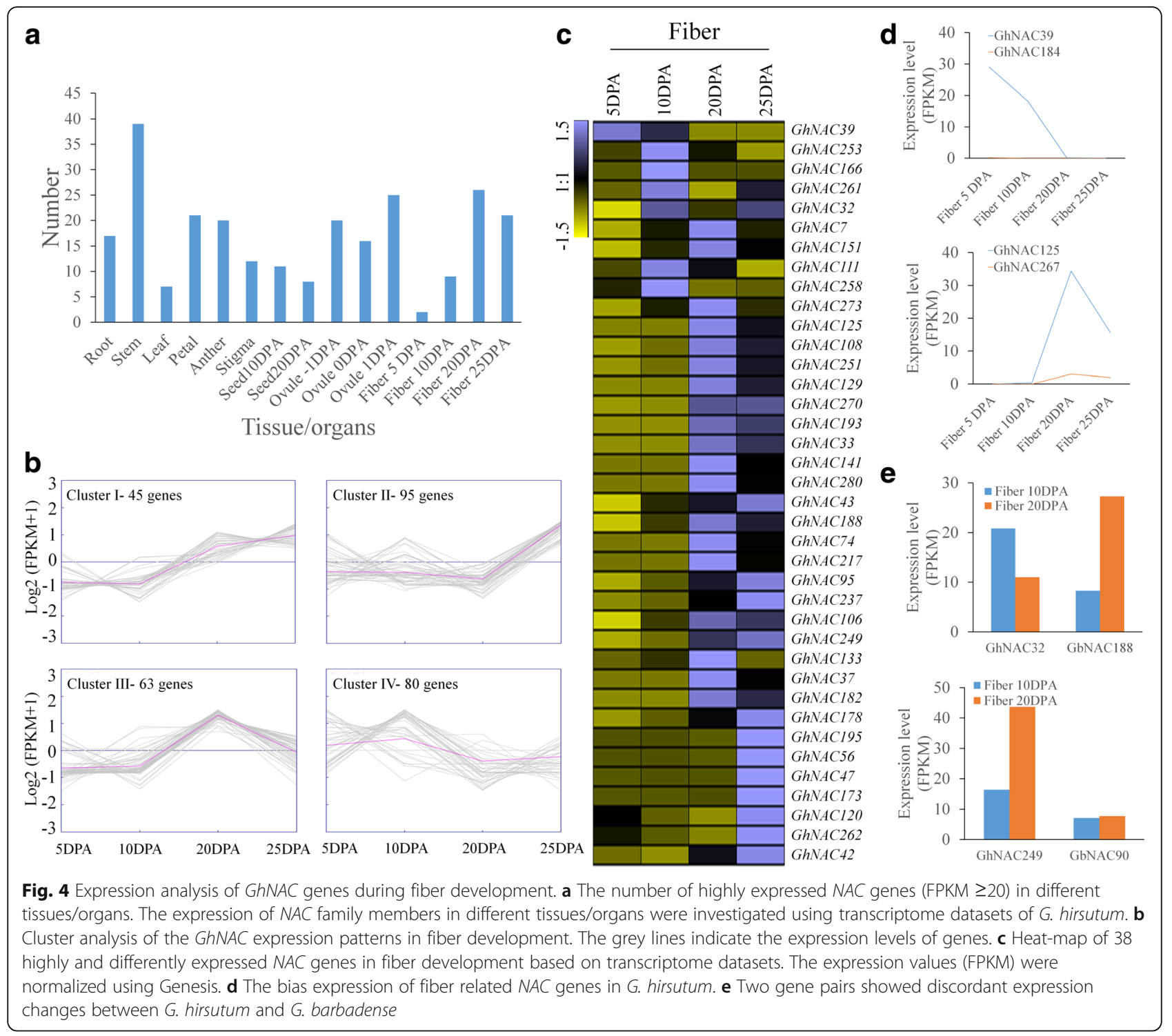

homologous gene expression patterns for the 38 GhNAC genes in three other cotton species, using transcriptome data sets for fiber development (10DPA and 20DPA) (Additional file 9: Figure S4b). Most of the genes showed similar expression changes, suggesting the conservation of functions of NAC genes in regulating fiber development between diploid and allotetraploid cotton. We also identified two gene pairs (GhNAC32 and GbNAC188, Gh NAC249 and GbNAC90) that showed significantly different expression patterns between Upland cotton G. hirsutum and extra-long staple cotton G. barbadense (Fig. 4e).

Six GhNAC genes were selected to verify the expression changes during fiber development progress $(0,5$, $10,20,25 \mathrm{DPA})$ by quantitative reverse-transcription -PCR (qRT-PCR) (Fig. 5a). All the selected genes showed almost the similar expression changes compared with transcriptome data sets. The expression of five highly expressed genes (GhNAC37, GhNAC125, GhNAC193, GhNAC273, GhNAC280), which were selected according to transcriptome data sets, showed sharp increases at the SCW thickening stage.

Four fiber development related NAC genes (GhNAC33, GhNAC125, GhNAC193, GhNAC280) were selected for co-expression analysis. Two hundred and ninety-one co-expressed genes were identified and some showed overlap with these four genes (Fig. 5b, Additional file 11: Table S7). Gene ontology analysis of the co-expressed genes showed that plant cell wall biogenesis was the most abundant functional term, and we identified cellulose synthase family genes with co-expression relationships, such as GhCESA7 (Gh_D07G0380), GhCESA4 (Gh_A0 7G1871), GhCESA8 (Gh_D10G0333). This suggests that $N A C$ genes might be involved in fiber development by regulating secondary cell wall synthesis (Fig. 5c). 


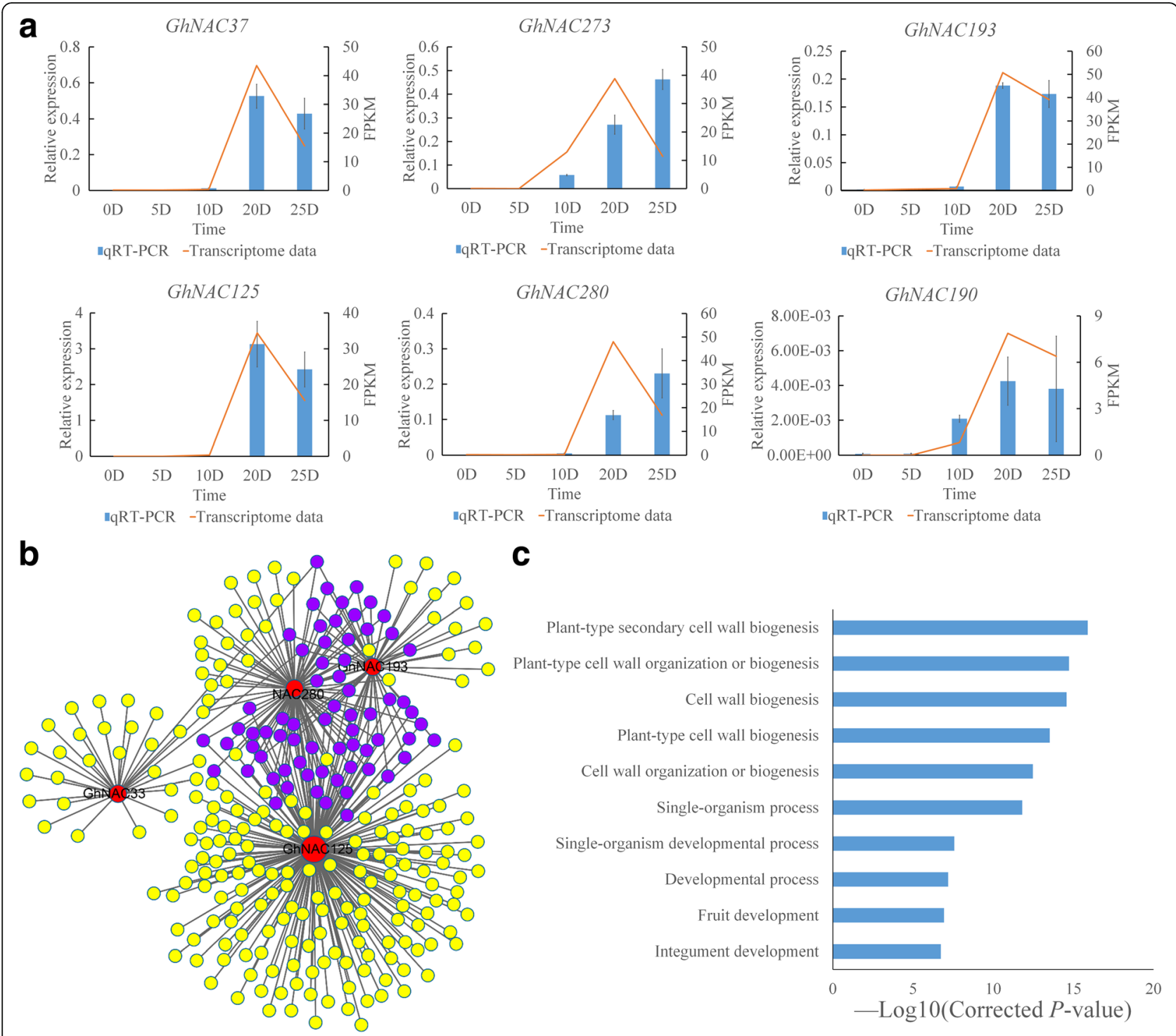

Fig. 5 The qRT-PCR and co-expression network analysis of fiber-related GhNAC genes. a Expression analysis of the selected GhNAC genes in fiber development by qRT-PCR. The GhUBQ7 (GenBank accession number: DQ116441) was used as the internal control to calculate and normalize the expression levels. Bars represent means \pm standard error $(n=3)$. The orange lines represent the transcriptome data (FPKM). b The co-expression network with gene expression view of GhNAC genes in fiber development. The gray lines between two nodes indicate co-expression relationships. The purple solid circles represent overlapped genes. c GO enrichment analysis of co-expression genes with GhNAC33, GhNAC125, GhNAC193, GhNAC280

\section{Identification of stress-related NAC genes in G. hirsutum} $N A C$ genes have received much attention as important regulators in various stress signaling pathways. We used the public transcriptome data sets of G. hirsutum treated with high temperature (heat), salinity, cold and polyethylene glycol (PEG) to investigate the expression patterns of GhNAC genes under abiotic stress (Additional file 12: Figure S5a). One hundred and twenty-four (43.8\%) GhNAC genes expressed at least in one stress $(F P K M \geq 1)$ and 120 (42.4\%) of these genes with two-fold different expression compared with controls in at least one treatment (Fig. 6a, Additional file 10: Table S6). There were 101, 73, 86 and
52 differentially expressed GhNAC genes were identified in heat, salinity, cold and PEG stress treatments, respectively. Comparative analysis showed that 29 GhNAC genes exhibited overlapping differential expression under the four stress conditions (Fig. 6b). Heat and cold had the most overlapping genes (70 genes), whereas cold and PEG stress had the least (39 genes). We also identified 10 stress-related GhNAC genes (GhNAC9, GhNAC15, Gh NAC34, GhNAC47, GhNAC56, GhNAC149, GhNAC158, GhNAC173, GhNAC192, GhNAC195) that are homologous with stress-related $N A C$ genes in Arabidopsis and rice, such as RD26, ANAC019, SNAC1 and SNAC2, which 


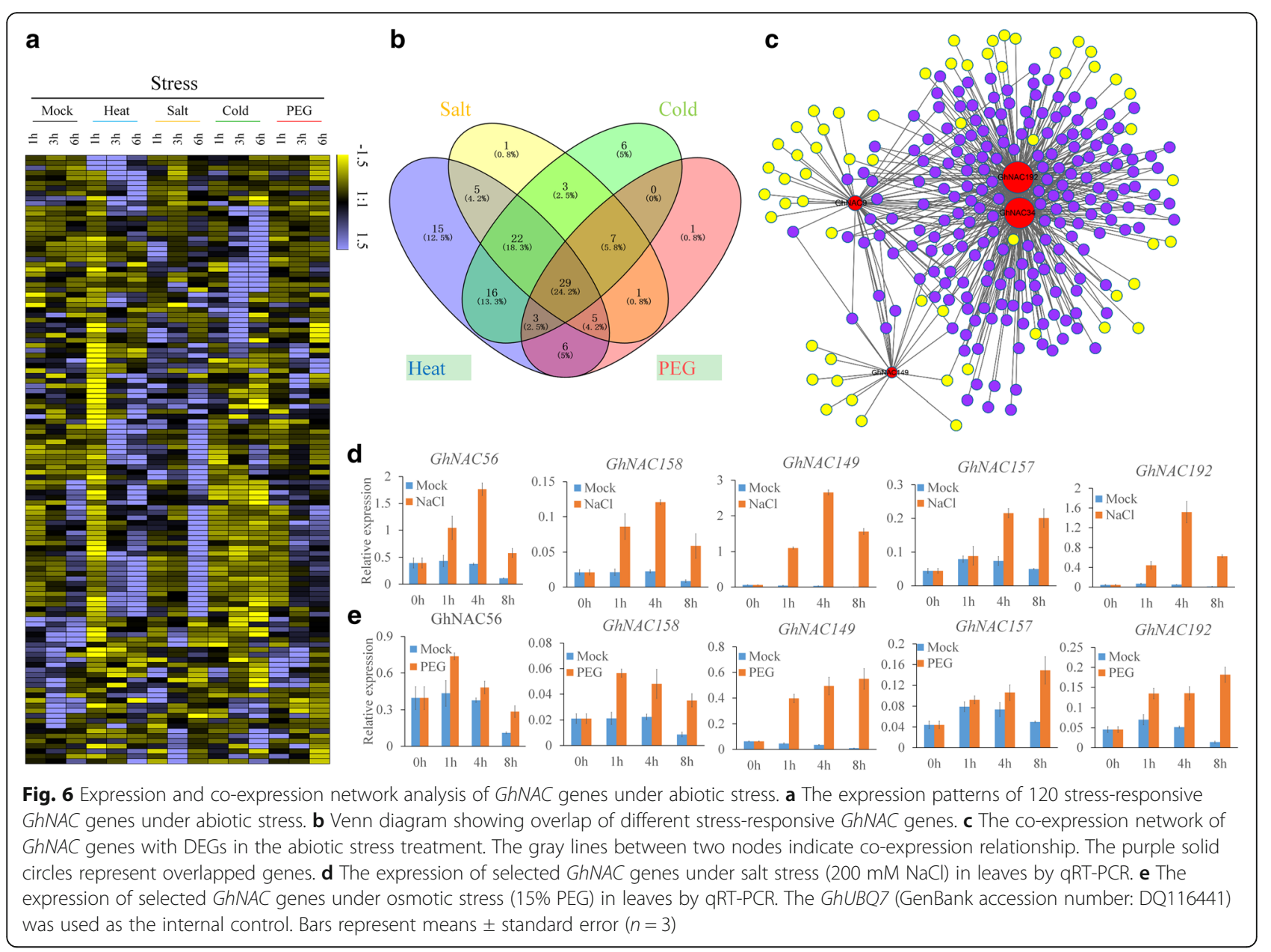

are reported to be involved in multiple stress responses (Additional file 12: Figure S5b). The expression patterns were closely related to evolutionary relationships. Our results indicate that some GhNAC genes might play conserved functions in stress responses in plants.

Four stress-related GhNAC genes (GhNAC9, GhNAC34, GhNAC149, GhNAC192) were selected for co-expressed network analysis. There were 58, 207, 22 and 227 co-expressed differentially expressed genes (DEGs) detected in GhNAC9, GhNAC34, GhNAC149 and Gh NAC192, respectively (Fig. 6c, Additional file 11: Table S7). Some co-expression genes were associated with stress responses, such as late embryogenesis abundant protein genes (Gh_D10G0248, Gh_D11G0978, Gh_D11G2003), heat shock transcription factor genes (Gh_D05G0228, Gh_D05G0307), peroxidase superfamily protein genes (Gh_D04G0130, Gh_A09G1575, Gh_A10G1317) and phytohormone responsive genes (Gh_A05G0278, Gh_ A05G0782, Gh_A05G3589). The 267 specific co-expression genes were annotated based on functional gene ontology using KOBAS 3.0, and some major enrichment categories were identified: response to acid chemical, response to endogenous stimulus, response to water deprivation (Additional file 12: Figure S5c).

The expression profiles of some stress-related GhNAC genes were verified by qRT-PCR under $200 \mathrm{mM}$ $\mathrm{NaCl}$, simulating salt stress; and 15\% PEG, simulating osmotic stress (Fig. 6d, e). The qRT-PCR results are consistent with transcriptome data sets, showing that the selected GhNAC genes are significantly induced by these two stresses. However, it is worth noting that these selected $N A C$ genes are barely induced by ABA treatment, with the exception of GhNAC164 (Additional file 13: Figure S6).

\section{Transactivation assay of GhNAC proteins}

Transcriptional activation is an important characteristic of NAC transcription factors. To investigate the transactivation capacity of NAC proteins in cotton, we performed a protoplast transient expression system, which based on the interaction between the DNA binding domain of GAL4 (GAL4DB) and the binding sites of GAL4(5X)-TATA-LUC. The full-length cDNA of selected GhNAC genes (stress related: GhNAC56, GhNAC149, GhNAC157, GhNAC158, 
GhNAC192; fiber development related: GhNAC37, GhNAC125, GhNAC193, GhNAC280) were cloned into the vector GAL4DB respectively. Results showed that in all cases the luciferase (LUC)/Renilla LUC ratio of GAL4DB-GhNACs was to a greater extent higher than that of control GAL4DB, and the membrane-bound NAC transcription factor, GhNAC157, had the strongest transcriptional activity (Fig. 7a). We also performed transactivation assays in the yeast strain $\mathrm{Y} 2 \mathrm{H}$, with the transactivation capacity determined by $\mathrm{X}-\alpha-\mathrm{Gal}$ (5-bromo-4-chloro-3-indoxyl a-D-galactoside) activity. Almost all selected NAC proteins had transactivation activity (blue colonies), except for GhNAC280 (Fig. 7b).

Furthermore, GhNAC157 (with TM) and GhNAC158 (without TM) were selected to investigate the transactivation activity domain. Our results showed that the C-terminal activation domain (AD) of GhNAC157 and GhNAC158 exhibited transactivation activity (Fig. 7c, d), which is consistent with previous studies. In addition, we found the TM domain of GhNAC157 in the C-terminal region did not affect the activation ability (Fig. 7d).

\section{Discussion}

Transcription factors operate as important switches of transcription networks and regulate gene expression accurately [45]. The NAC acronym is derived from NAM (no apical meristem), ATAF1/2, and CUC2 (cup-shaped cotyledon), three genes which were initially discovered to contain a conserved NAC domain $[4,17,18]$. In cotton, we found subdomains $\mathrm{C}$ and $\mathrm{D}$ with the highly conserved and positively charged amino-acid residues, and these two subdomains might be associated with DNA binding [5]. More recent research has shown that the $N A C$ genes constitute one of the largest plant-specific transcription factor families, expanding during the evolution in land plants, possibly associated with the elaborate developmental program critical to maximize plant fitness [46].

In the present study, we took advantage of the available genome data of four sequenced cotton species, and the $N A C$ genes were identified located throughout these genomes. Unlike the traditional identification methods which align the conserved NAC domains, we identified NAC family genes based on the whole coding genes of four cotton species. Our results show that about $0.35 \% \sim 0.4 \%$ genes encode NAC transcription factors in the cotton genome, with the NAC genes representing 5.5\% 5.7\% of all transcription factor genes. The $N A C$ gene numbers which were identified in our study are different compared with previous reports, which might be caused by the difference of the reference genomes or the identification methods [36, 43, 44]. Phylogenetic and gene homology analyses discovered the evolutionary relationship between four cotton species, and the extensive homology of $N A C$ genes between diploid and tetraploid cotton is consistent with a previous study that shows tetraploid cotton species $(\mathrm{AD})$ is derived from an interspecific hybridization between an A-genome ancestral species, G. herbaceum (A1) or G. arboreum (A2) and a native D-genome species, G. raimondii (D5) or G. gossypioides (D6) [47]. However, the inconsistent number of homologous gene pairs indicates the independent evolution of each subgenome pairs.

There are extensive variations in gene length, predicted protein molecular weight and protein isoelectric

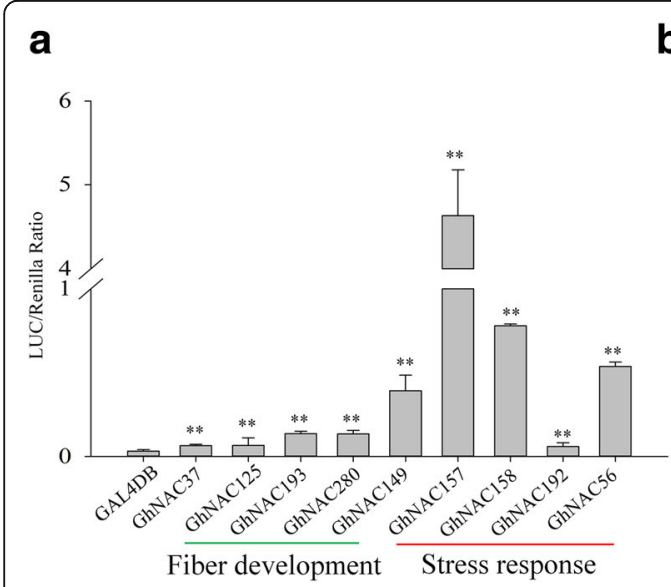

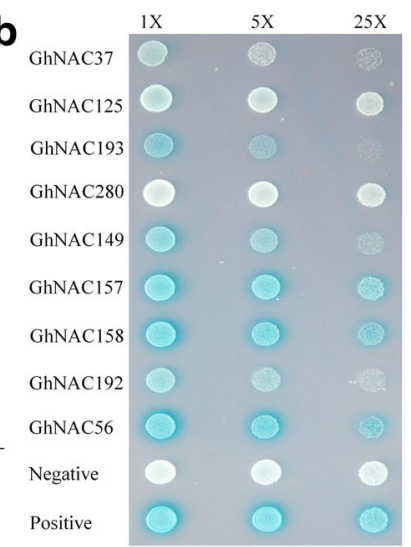

Positive

Fiber development Stress response

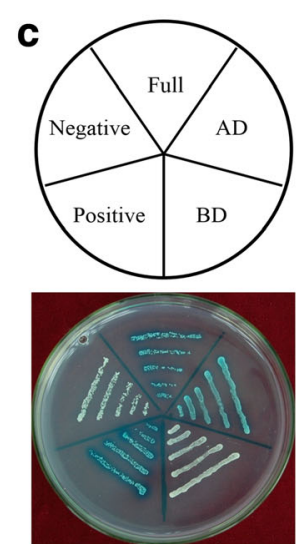

GhNAC158

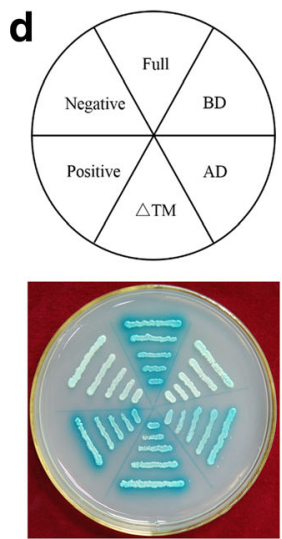

GhNAC157

Fig. 7 Transactivation analysis of GhNAC genes. a The transcriptional activity of selected NAC proteins in cotton embryogenic callus protoplasts. Renilla LUC was used as the internal control. Three independent experiments were performed. Asterisks indicate significant differences, $* * *$ 0.01 , student's t-test. The LUC/Renilla Ratio of each gene compared with control (GAL4DB), respectively. $\mathbf{b}$ Transactivation assay of NAC proteins in yeast strain $\mathrm{Y} 2 \mathrm{H}$. The transactivation activities were determined by the growth on SD medium without Tryptophane (Trp) and added X-a-Gal (SD-Trp + X-a-Gal). The photograph was taken after $36 \mathrm{~h}$ incubation. Positive control: pGBKT7-53; negative control: pGBKT7-Lam. c Transactivation assay of GhNAC158. Full: the full length of GhNAC158, BD: NAC domain (Binding Domain) of GhNAC158, AD: the C terminal (Activation Domain) of GhNAC158. d Transactivation assay of GhNAC157. $\triangle \mathrm{TM}$ : the transmembrane motif (TM) deleted form of GhNAC157 
point, whereas the gene structures are relatively conserved in the NAC gene family, with about $68.2 \%$ GhNAC genes having three exons (Fig. 2a). In particular, a high degree of similarity was detected in gene structure and predicted protein properties between homologous gene pairs. This result proves that duplicate genes originating from the progenitors can evolve independently at the same rate and show few changes [48].

Membrane-bound transcription factors (MTF) are located in the membrane itself and are activated upon receiving of specific signal, and this regulation mode is considered to be accurate, such as membrane release [10]. Membrane-bound NAC transcription factors have been extensively identified and involved in many biological processes in different plant species $[9,11,49,50]$. In this study, we firstly identified 68 predicted membrane-bound NAC transcription factors in four cotton species and members are mainly distributed in the same subfamily, and these genes appear to be conserved during evolution. Except for the classical structures (with one $\alpha$-helical TM located in the C terminal), there are some divergent forms also identified, such as two genes possessing two TM, and TM preceding the NAC domain. These forms were also identified in tomato, however, this was not found in Arabidopsis and rice, and the inference is that these divergent $N A C$ genes have evolved functional specificity. Phylogenetic analysis showed some clades contained membrane-bound NAC genes derived from all of the monocots (rice) and dicots (cotton, tomato and Arabidopsis), suggesting that these genes probably shared a common ancestor that predated the divergence of the monocots and dicots (Additional file 4: Figure S2d).

Upland cotton (G. hirsutum) is a model for studying the domestication of polyploid crops. In this study, there are higher SNP densities in GhNAC genes in wild cotton than that in cultivated cotton, and it is suggested that the evolution of Upland cotton was accompanied by selection and domestication (Fig. 2c). There is a higher variant range of the A subgenome than of the D subgenome in wild cotton, consistent with the previous study that Upland cotton revealed asymmetric evolution between the A and D subgenomes [42]. For selective pressure analysis, we found purifying selection acted as a primary force in the evolution of NAC genes in cotton, therefore, they might retain their ancestral functions [11].

Cotton fiber is the most important natural textile material in the world, and the improvement of fiber quality is the major goal of cotton breeding. At $16 \mathrm{~d}$ post anthesis, the SCW thickening stage initiates and cellulose synthesis is at a high rate, and is the main constituent (> 90\%) of the mature fibers [32]. Previous studies showed there are 24 and 14 NAC genes which exhibit higher expression in 15 DPA fibers in G. arboreum and G. raimondii, respectively [44]. Here, we identified 38 GhNAC genes showing high and differential expression during fiber development, with most genes preferentially expressed at the secondary cell wall deposition stage, suggesting $N A C$ genes might play an important role in this process (Fig. 4c). NAC transcription factors participating in SCW formation have been well reported, and the roles of SCW-related $N A C$ genes are conserved in different plant species [13, 19, 23, 51]. Phylogenetic analysis revealed some GhNAC genes show homology with the SCW biosynthesis related NAC gene NST1 (ANAC043) of Arabidopsis. Combined with previous studies, we suggest that $N A C$ genes regulating cotton fiber development might do so through the regulation of secondary cell wall formation. Consistent with our hypothesis, a recent study showed that a cotton NAC gene, GhFSN1, regulates fiber quality by promoting SCW biosynthesis [25]. GhFSN1 is a fiber-related candidate gene (GhNAC125) in our study. Our results show that co-expression relationships exist between GhNAC125 and genes involved plant-type secondary cell wall biogenesis, such as GhCESA8 (CELLULOSE SYNTHASE 8, Gh_D10G0333) and GhCOBL4 (COBRA-LIKE4, Gh_D03G0919) (Additional file 11: Table S7). We also found that the homologous gene of GhFSN1 (Gh $N A C 125)$, GhNAC267, shows low expression in fiber, and this indicates the functional differentiation of homologous gene pairs between A subgenome and D subgenome (Fig. 4d). The comprehensive analysis of NAC genes in fiber development will prove instructive for their function in this developmental context.

Nowadays, more and more cotton cultivation areas are concentrated in marginal lands, which are threatened frequently by extreme environmental conditions, and these stresses will inevitably affect the growth, productivity, and fiber quality [52]. Much work has been performed in the past two decades to reveal the significance of NAC transcription factors in regulating various stress signaling pathways $[5,26,53]$. The study of the mechanism of cotton responses to abiotic stress has proved difficult, and so identifying valuable genes for cotton molecular breeding is necessary. Previous genomic sequencing has revealed that $N A C$ genes may regulate abiotic stress tolerance in cotton [42]. Although some NAC genes involved in stress response have been identified, research on the biological functions and mechanisms is still in its infancy in cotton $[35,53,54]$. There are 33 NAC genes that respond to salt stress in Arabidopsis, and $40 N A C$ genes change significantly in rice under drought or salt stress $[55,56]$. Here, we identified 120 (42.4\%) abiotic stress-responsive GhNAC genes, and 29 genes showed differential expression under four stress conditions, these genes might be involved in the regulation of various stresses (Fig. 6). Most stress-responsive 
GhNAC genes changed significantly under heat and cold stresses, suggesting they might have important biological functions in response to temperature stress. It is noteworthy that the expression of some GhNAC genes is clustered with the stress response NAC genes of Arabidopsis and rice, and we speculate that these genes might play conserved functions in stress responses across species (Additional file 12: Fig. S5b). Previous studies partly show the validity of our results. For example, GhATAF1 (GhNAC173) is highly induced by salt stress, and transgenic cotton plants overexpressing GhATAF1 show enhanced tolerance to salt stress, by up-regulating the expression of stress-related genes and decreasing the $\mathrm{Na}^{+}$content in shoots [34]. Some stress-responsive GhNAC genes show differential expression patterns induced by ABA treatment, and it is implied that some GhNAC genes could be regulated by ABA-dependent and ABA-independent pathways. In addition, 27 common GhNAC genes showed differential expression under stress responses and fiber development, indicating these genes may play multiple functions in cotton (Fig. 8). The stress-responsive GhNAC genes identified in this study could be used as promising candidates for molecular breeding to create new cotton varieties possessing good agronomic traits under adverse conditions.

\section{Conclusion}

At present, improving the fiber quality and stress tolerance are the major goals for cotton breeding. The largest plant-specific NAC TFs are renowned for their roles in diverse developmental programs. We have comprehensively identified the NAC genes in four cotton species. Fiber development and stress response related GhNAC genes were highlighted for their expression patterns, co-expression network and transactivation (Fig. 8) This result will provide useful insights on $N A C$ genes for further genetic improvement in cotton. However, it should be noted that the present analysis represents a starting point for functional studies of GhNAC genes on fiber development and stress response, and further experimental researches are required to expand on the findings

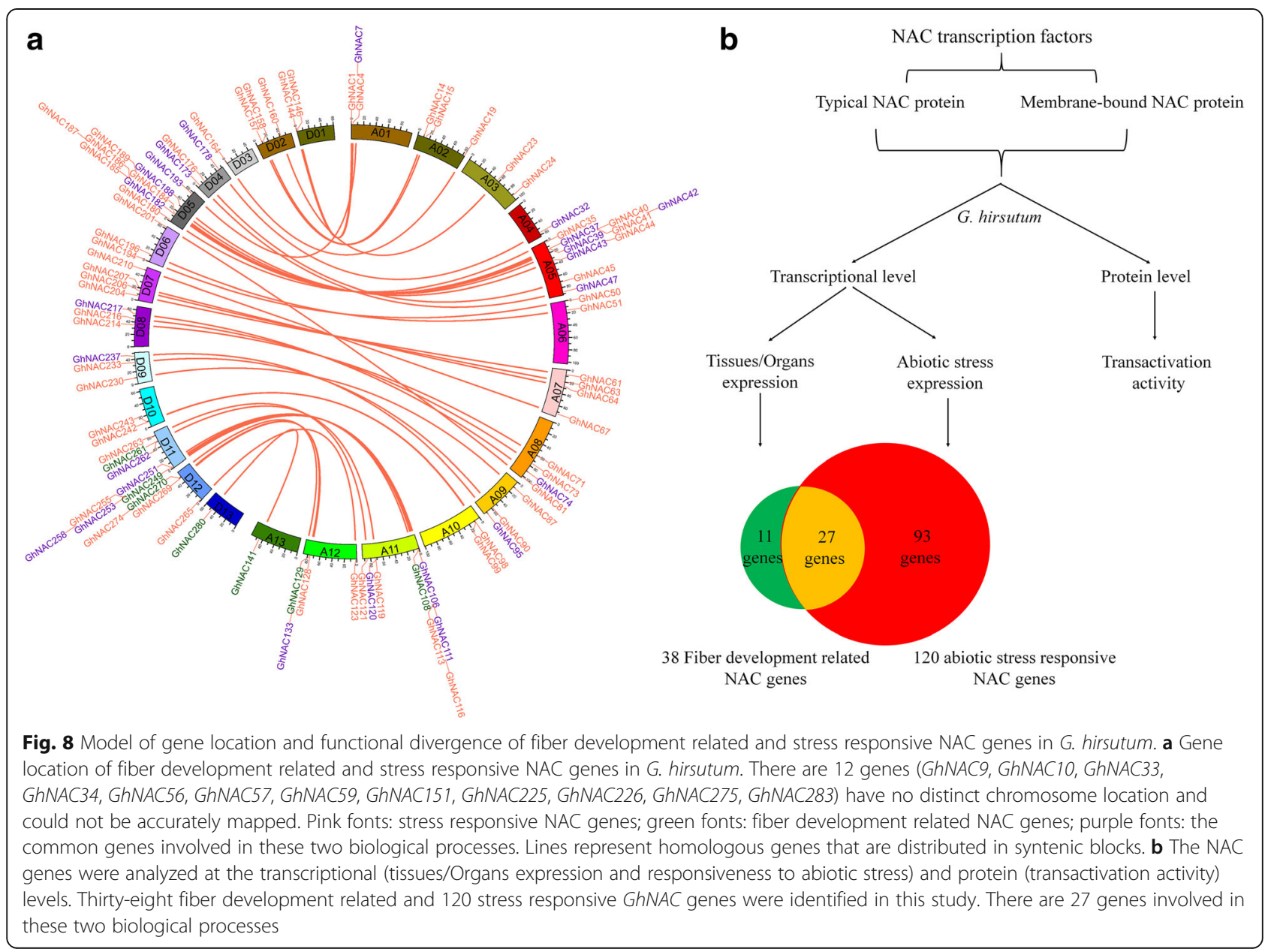


obtained to define the biological function and molecular mechanisms of the highlighted GhNAC genes.

\section{Methods \\ Identification and phylogenetic analysis of the NAC family genes in Gossypium spp}

To identify $N A C$ genes in four cotton species, we downloaded all the predicted protein sequences from CottonFGD (https://cottonfgd.org/about/download.html) [57]. NAC genes were identified by PlantTFDB 4.0 (http:// planttfdb.cbi.pku.edu.cn/). In addition, complete aminoacid sequences were analyzed using ClustalX (ver.1.83) and MEME (http://meme-suite.org/index.html) to discover conserved motifs of NAC genes to conform our identification. The predicted molecular weight and isoelectric points of NAC proteins were calculated using the ExPASy program (http://web.expasy.org/protparam/). The membrane-bound NAC members were identified using the TMHMM server v.2.0 (http://www.cbs.dtu.dk/services/TMHMM/).

Multiple sequence alignments of the protein sequences were carried out using ClustalX (ver.1.83) with default settings. The conserved sequence logo of NAC domains (A-E) were generated using MEME (http://meme-suite.org/index.html). The complete amino acid sequences of predicted NAC proteins were used for phylogenetic analysis. Phylogenetic trees were constructed using MEGA7 software by the Neighbour-Joining method [58]. The evolutionary distances were computed using the Poisson correction method and the nodes of the trees were evaluated by boot-strap analysis with 1000 re plicates.

\section{Gene structure, promoter, genome synteny and variation analysis of GhNAC genes}

To analyze gene structures, the coding and genomic sequences of GhNAC genes were downloaded and analyzed by the online Gene Structure Display Server (GSDS2.0) program (http://gsds.cbi.pku.edu.cn/). About 1.5-kb upstream regions from the initiation codon of each GhNAC genes were analyzed by PlantCARE database (http://bioinformatics.psb.ugent.be/webtools/plant$\mathrm{care} / \mathrm{html} /$ ) to reveal promoter cis-elements [59].

Genome synteny and variation analysis were performed as described previously [60]. The At and Dt, At and A2, Dt and D5 reciprocal aligned sequences were obtained using BLASTN (e value <1e-05). We used Circos plot to show these homologous gene pairs.

For molecular evolutionary properties, we calculated the non-synonymous $\left(\mathrm{d}_{\mathrm{N}}\right)$ and synonymous $\left(\mathrm{d}_{\mathrm{S}}\right)$ substitution rates within and between cotton species to explore the evolutionary dynamics and selection pressures [61]. The ratio $d_{N} / d_{S}>1,=1$ and $<1$ indicating positive (or diversifying) selection, neutral evolution, and purifying (or negative) selection, respectively. For molecular evolutionary properties analysis, the ratio of non-synonymous to synonymous for the homologous gene pairs were calculated by Maximum Likelihood (PAML) yn00 program with the GMYN method.

For variation analysis of $N A C$ genes, our previous genome data from 31 wild cotton and 321 domesticated Upland cotton were used to calculate the SNP densities as previously described [60, 62].

\section{Expression profiles and co-expression networks analysis}

The transcriptome data sets corresponding to expression abundances of TM-1 (The allotetraploid cotton G. hirsutum L. acc. Texas Marker-1) in different tissues and stresses from NCBI were used to analysis the expression profiles of GhNAC genes (https://www.ncbi.nlm.nih.gov/ sra/?term = PRJNA248163) and CottonFGD (https://cottonfgd.org/) [42, 57]. The gene expression patterns were showed by heatmap with the expression values normalized by Genesis software [63]. The expression change of $N A C$ genes during fiber development were clustered by the Genesis K-means method [63].

The co-expression networks analysis of GhNACs involved in fiber development and stress response were performed as described previously with slight modification [60]. Pearson correlation coefficient (PCC) was used to measure the co-expression relationships between the selected GhNAC genes and co-expression genes. For fiber analysis, fourteen published RNA-Seq data sets were obtained, including ovule and fiber development. Weighted gene co-expression network (WGCNA) analysis was performed as described previously [64]. Genes with $\mathrm{PCC}(|\mathrm{PCC}| \geq 0.8$ for stress, $|\mathrm{PCC}| \geq 0.9$ for fiber $)$ were used for constructing co-expression networks. All of the co-expression networks were visualized by Cytoscape software (3.4.0). The co-expression genes were annotated based on functional gene ontology using KOBAS 3.0 [65].

\section{Plant materials and treatments}

Gossypium hirsutum cv. YZ1 were cultivated in Hoagland solution in $28{ }^{\circ} \mathrm{C}$ culture room with $16 \mathrm{~h}$ light $/ 8 \mathrm{~h}$ dark photoperiod cycle conditions. Four-week-old seedlings were treated with Hoagland solution containing $200 \mathrm{mM} \mathrm{NaCl}, 15 \%$ PEG, and $0.5 \mu \mathrm{M}$ ABA, respectively. The leaves and roots were collected at different time intervals, immersed in liquid nitrogen immediately and then frozen at $-80{ }^{\circ} \mathrm{C}$ for later use. To test the expression of selected genes in different tissues/organs, the methods for tissue samples collection were performed as previously described [66].

\section{qRT-PCR analysis}

qRT-PCR analysis was performed as described previously with little modification [66]. High-quality RNA 
was reverse transcribed to cDNA using SuperScript III Reverse Transcriptase in accordance with the manufacturer's instructions (Cat. No.18080-093, Invitrogen). qRT-PCR experiments were performed using an ABI Prism 7500 system (Applied Biosystems) and the comparative $\mathrm{Ct}\left(2^{-\Delta \mathrm{Ct}}\right)$ method was used to calculate gene expression levels. GhUBQ7 (GenBank accession No.DQ116441) was used as the internal control. Gene-specific primers for qRT-PCR were designed according to the cDNA sequences using Primer Premier 5.0 software and synthesized commercially (Genscript Bioscience). The primers used for qRT-PCR are listed in Additional file 14: Table S8.

\section{Transcriptional activation analysis}

The transactivation activation ability of NAC proteins was performed by protoplast transient expression system as previously described [66]. Reporter construct contained five copies of the GAL4 binding site in tandem and a minimal TATA region of the CaMV $35 \mathrm{~S}$ promoter, the firefly gene for luciferase (LUC). Selected NAC genes were cloned into the vector GAL4DB and transformed into Escherichia coli TOP 10. After culturing, the plasmids were extracted and transferred to protoplast derived from cotton embryogenic calluses using PEG-calcium transformation method as described previously [67]. A dual-luciferase assay was used to detect the LUC and Renilla LUC (Promega, Cat. no. E1910).

For yeast transactivation assay, a bait protein is expressed as a fusion to the Gal4 DNA-binding domain (DNA-BD), the gene coding sequences were cloned respectively into the pGBKT7 vector to achieve this goal. Here, the full-length coding sequences of GhNAC genes and the truncated forms (GhNAC157-BD, GhNAC157-AD, GhNAC157 TM, GhNAC158-BD, GhNAC158-AD) were cloned respectively into the pGBKT7 vector. These vectors were transformed into $\mathrm{Y} 2 \mathrm{H}$ gold strains according to the instruction manual (Cat. No.630489, Clontech), and transactivation activity was determined by the growth on SD medium without Trp and added X- $\alpha-G$ al (SD-Trp $+\mathrm{X}-\alpha-\mathrm{Gal})$. The photograph was taken after $36 \mathrm{~h}$ incubation. The primers used in the transactivation assay are listed in Additional file 14: Table S8.

\section{Additional files}

Additional file 1: Table S1. Protein sequences and nomenclature of cotton NAC genes. (XLSX $166 \mathrm{~kb}$ )

Additional file 2: Figure S1. Statistical analysis of sequence length, molecular weight and isoelectric point for NAC protein in cotton. (TIF $478 \mathrm{~kb}$ )

Additional file 3: Table S2. Basic characteristic of NAC genes in cotton. (XLSX $54 \mathrm{~kb}$ )
Additional file 4: Figure S2. The deviant structures of membranebound NAC genes in cotton. (a) GhNAC157 had a TM at C terminal. (b) GaNAC111 possessed two TM at C terminal. (c) NAC genes with TM preceded the conserved NAC domain. (d) Phylogenetic analysis of membrane-bound NAC transcription factors between cotton and other plant species. Phylogenetic tree was made by MEGA7 software through the Neighbour-Joining method. G. hirsutum (red square), G. barbadense (green square), G. raimondii (blue triangle), G. arboreum (magenta triangle), Arabidopsis (blue hollow square), Oryza sativa (magenta hollow triangle), Solanum lycopersicum (black hollow triangle). (TIF $3906 \mathrm{~kb}$ )

Additional file 5: Figure S3. The inter-genomic (At and Dt) synteny analysis of NAC genes. Lines represent homologous genes that are distributed in syntenic blocks. The 't' indicates tetraploid. (TIF $4880 \mathrm{~kb}$ )

Additional file 6: Table S3. The chromosome location of NAC genes in G. hirsutum. (XLSX $25 \mathrm{~kb})$

Additional file 7: Table S4. The homologous gene pairs were identified in cotton. (XLSX $14 \mathrm{~kb}$ )

Additional file 8: Table S5. Cis-element analysis of NAC genes in G. hirsutum. (XLSX $34 \mathrm{~kb}$ )

Additional file 9: Figure S4. The expression patterns of NAC genes. (a) The expression patterns of GhNAC genes in different tissues/organs. (b) The expression patterns for homologous genes of the 38 highly expressed GhNAC genes during 10 and 20 DPA fiber development in four cotton species. (TIF $4910 \mathrm{~kb}$ )

Additional file 10: Table S6. The transcriptome data for fiber development and stress responsive GhNAC genes. (XLSX $102 \mathrm{~kb}$ )

Additional file 11: Table S7. Co-expression genes with selected NAC genes involved in fiber development and stress response. (XLSX $31 \mathrm{~kb}$ )

Additional file 12: Figure $\mathbf{S 5}$. The expression analysis of stress-related GhNAC genes. (a) The expression patterns of GhNAC genes under abiotic stress. (b) Phylogenetic relationship of GhNAC proteins with previously reported stress related NAC proteins in Arabidopsis and rice. ANAC019 (AT1G52890), ANAC055 (AT3G15500), RD26 (AT4G27410), ANAC002 (AT1G01720), SNAC1 (Os03g0815100), SNAC2 (XP_015620920). (c) GO enrichment analysis of co-expression genes. (TIF $2882 \mathrm{~kb}$ )

Additional file 13: Figure S6. The expression of selected GhNAC genes under ABA $(0.5 \mu \mathrm{M})$ treatment. The GhUBQ7 (GenBank accession number: DQ116441) was used as the internal control. Bars represent means \pm standard error $(n=3)$. (TIF $503 \mathrm{~kb})$

Additional file 14: Table S8. Primer sequences were used in this study. (XLSX $11 \mathrm{~kb})$

\section{Abbreviations}

A2: A-genome species; ABA: Abscisic acid; At: A subgenome, ' $t$ ' denoting tetraploid; BD: DNA-binding domains; D5: D-genome species;

DEG: Differentially expressed genes; $d_{N}$ : Non-synonymous; DPA: Days post anthesis; $d_{s}$ : Synonymous; Dt: D subgenome; HSE: Heat stress response element; LUC: Luciferase; MTF: Membrane-bound transcription factors; NAC: NAM, ATAF, and CUC; PEG: Polyethylene glycol; SCW: Secondary cell wall; SNP: Single nucleōtide polymorphism; TM: Transmembrane motif; TR: Transcriptional regulatory regions; X-a-Gal: 5-bromo-4-chloro-3-indoxyl aD-galactoside

\section{Acknowledgements}

We are grateful to Keith Lindsey (Integrative Cell Biology Laboratory, School of Biological and Biomedical Sciences, University of Durham) for the modification of article language.

\section{Funding}

This work was supported by funding from the National Key Project of Research and the Development Plan (2016YFD0101006) and the Fundamental Research Funds for the Central Universities (2662016PY001).

Availability of data and materials

The nucleotide and protein sequences of genes identified in this paper could be downloaded from Cotton Functional Genomics Database (CottonFGD) (https://cottonfgd.org/). The transcriptome data sets of TM-1 in 
different tissues and stresses were downloaded from NCBI (https:// www.ncbi.nlm.nih.gov/ sra/?term = PRJNA248163) and CottonFGD. The datasets used during the current study are available from the corresponding author on reasonable request.

\section{Authors' contributions}

$H S, X Y$ and $X Z$ conceived and designed the experiments. HS, MH, JL and LC performed the experiments. SH, ML and SZ discussed and commented on the content of the article. HS wrote the article. XY supervised and complemented the article. All authors read and approved the final manuscript.

\section{Ethics approval and consent to participate} Not applicable.

\section{Consent for publication}

Not applicable.

\section{Competing interests}

The authors declare that they have no competing interests.

\section{Publisher's Note}

Springer Nature remains neutral with regard to jurisdictional claims in published maps and institutional affiliations.

\section{Received: 8 April 2018 Accepted: 17 July 2018}

\section{Published online: 24 July 2018}

\section{References}

1. Le DT, Nishiyama R, Watanabe Y, Mochida K, Yamaguchi-Shinozaki K, Shinozaki K, Tran LS. Genome-wide survey and expression analysis of the plant-specific NAC transcription factor family in soybean during development and dehydration stress. DNA Res. 2011;18(4):263-76.

2. Nuruzzaman M, Manimekalai R, Sharoni AM, Satoh K, Kondoh H, Ooka H, Kikuchi S. Genome-wide analysis of NAC transcription factor family in rice. Gene. 2010:465(1-2):30-44

3. Yan H, Zhang A, Ye Y, Xu B, Chen J, He X, Wang C, Zhou S, Zhang X, Peng $Y$, et al. Genome-wide survey of switchgrass NACs family provides new insights into motif and structure arrangements and reveals stress-related and tissue-specific NACs. Sci Rep. 2017;7(1):3056.

4. Ooka H, Satoh K, Doi K, Nagata T, Otomo Y, Murakami K, Matsubara K, Osato $\mathrm{N}$, Kawai J, Carninci P. Comprehensive analysis of NAC family genes in Oryza sativa and Arabidopsis thaliana. DNA Res. 2003;10(6):239-47.

5. Puranik S, Sahu PP, Srivastava PS, Prasad M. NAC proteins: regulation and role in stress tolerance. Trends Plant Sci. 2012;17(6):369-81.

6. Kim MJ, Park MJ, Seo PJ, Song JS, Kim HJ, Park CM. Controlled nuclear import of the transcription factor NTL6 reveals a cytoplasmic role of SnRK2.8 in the drought-stress response. Biochem J. 2012:448(3):353-63.

7. Lee S, Seo PJ, Lee HJ, Park CM. A NAC transcription factor NTL4 promotes reactive oxygen species production during drought-induced leaf senescence in Arabidopsis. Plant J. 2012;70(5):831-44.

8. Seo PJ, Kim MJ, Park JY, Kim SY, Jeon J, Lee YH, Kim J, Park CM. Cold activation of a plasma membrane-tethered NAC transcription factor induces a pathogen resistance response in Arabidopsis. Plant J. 2010;61(4):661-71.

9. Bhattacharjee P, Das R, Mandal A, Kundu P. Functional characterization of tomato membrane-bound NAC transcription factors. Plant Mol Biol. 2017; 93(4-5):511-32.

10. Kim SY, Kim SG, Kim YS, Seo PJ, Bae M, Yoon HK, Park CM. Exploring membrane-associated NAC transcription factors in Arabidopsis: implications for membrane biology in genome regulation. Nucleic Acids Res. 2007;35(1):203-13.

11. Li S, Wang N, Ji D, Xue Z, Yu Y, Jiang Y, Liu J, Liu Z, Xiang F. Evolutionary and functional analysis of membrane-bound NAC transcription factor genes in soybean. Plant Physiol. 2016:172(3):1804-20.

12. Sanggyu K, Sangmin L, Piljoon S, Soonkap K, Jeongkook K, Chungmo P. Genome-scale screening and molecular characterization of membranebound transcription factors in Arabidopsis and rice. Genomics. 2010; 95(1):56-65.

13. Nakano Y, Yamaguchi M, Endo H, Rejab NA, Ohtani M. NAC-MYB-based transcriptional regulation of secondary cell wall biosynthesis in land plants. Front Plant Sci. 2015;6:288
14. Nuruzzaman M, Sharoni AM, Kikuchi S. Roles of NAC transcription factors in the regulation of biotic and abiotic stress responses in plants. Front Microbiol. 2013:4:248.

15. Olsen AN, Ernst HA, Leggio LL, Skriver K. NAC transcription factors: structurally distinct, functionally diverse. Trends Plant Sci. 2005;10(2):79-87.

16. Wang Z, Dane F. NAC (NAM/ATAF/CUC) transcription factors in different stresses and their signaling pathway. Acta Physiol Plant. 2013;35(5):1397-408

17. Souer E, Van HA, Kloos D, Mol J, Koes R. The no apical meristem gene of Petunia is required for pattern formation in embryos and flowers and is expressed at meristem and primordia boundaries. Cell. 1996;85(2):159-70.

18. Aida M, Ishida T, Fukaki H, Fujisawa H, Tasaka M. Genes involved in organ separation in Arabidopsis: an analysis of the cup-shaped cotyledon mutant. Plant Cell. 1997;9(6):841-57.

19. Zhong $\mathrm{R}$, Demura $\mathrm{T}$, Ye ZH. SND1, a NAC domain transcription factor, is a key regulator of secondary wall synthesis in fibers of Arabidopsis. Plant Cell. 2006:18(11):3158-70

20. Zhong R, Richardson EA, Ye ZH. Two NAC domain transcription factors, SND1 and NST1, function redundantly in regulation of secondary wall synthesis in fibers of Arabidopsis. Planta. 2007;225(6):1603-11.

21. Kubo M, Udagawa M, Nishikubo N, Horiguchi G, Yamaguchi M, Ito J, Mimura T, Fukuda H, Demura T. Transcription switches for protoxylem and metaxylem vessel formation. Genes Dev. 2005;19(16):1855.

22. Zhou J, Zhong R, Ye ZH. Arabidopsis NAC domain proteins, VND1 to VND5, are transcriptional regulators of secondary wall biosynthesis in vessels. PLoS One. 2014;9(8):e105726.

23. Mitsuda N, Ohmetakagi M. NAC transcription factors NST1 and NST3 regulate pod shattering in a partially redundant manner by promoting secondary wall formation after the establishment of tissue identity. Plant J. 2008;56(5):768-78.

24. Xu B, Ohtani M, Yamaguchi M, Toyooka K, Wakazaki M, Sato M, Kubo M, Nakano Y, Sano R, Hiwatashi Y. Contribution of NAC transcription factors to plant adaptation to land. Science. 2014;343(6178):1505-8.

25. Zhang J, Huang GQ, Zou D, Yan JQ, Li Y, Hu S, Li XB. The cotton (Gossypium hirsutum) NAC transcription factor (FSN1) as a positive regulator participates in controlling secondary cell wall biosynthesis and modification of fibers. New Phytol. 2017;217(2):625-40.

26. Shao $\mathrm{H}$, Wang $\mathrm{H}$, Tang $\mathrm{X}$. NAC transcription factors in plant multiple abiotic stress responses: progress and prospects. Front Plant Sci. 2015;6:902.

27. Fujita M, Fujita Y, Maruyama K, Seki M, Hiratsu K, Ohme-Takagi M, Tran LS, Yamaguchi-Shinozaki K, Shinozaki K. A dehydration-induced NAC protein, RD26, is involved in a novel ABA-dependent stress-signaling pathway. Plant J. 2004;39(6):863-76

28. Tran LS, Nakashima K, Sakuma Y, Simpson SD, Fujita Y, Maruyama K, Fujita $M$, Seki M, Shinozaki K, Yamaguchi-Shinozaki K. Isolation and functional analysis of Arabidopsis stress-inducible NAC transcription factors that bind to a drought-responsive cis-element in the early responsive to dehydration stress 1 promoter. Plant Cell. 2004;16(9):2481-98

29. Sakuraba Y, Kim YS, Han SH, Lee BD, Paek NC. The Arabidopsis transcription factor NAC016 promotes drought stress responses by repressing AREB1 transcription through a trifurcate feed-forward regulatory loop involving NAP. Plant Cell. 2015;27(6):1771-87.

30. Hu H, Dai M, Yao J, Xiao B, Li X, Zhang Q, Xiong L. Overexpressing a NAM, ATAF, and CUC (NAC) transcription factor enhances drought resistance and salt tolerance in rice. Proc Natl Acad Sci. 2006:103(35):12987-92.

31. Fang $Y$, Liao $K, D u H, X u Y$, Song $H$, Li X, Xiong L. A stress-responsive NAC transcription factor SNAC3 confers heat and drought tolerance through modulation of reactive oxygen species in rice. J Exp Bot. 2015: 66(21):6803-17.

32. Haigler $\mathrm{CH}$, Betancur L, Stiff MR, Tuttle JR. Cotton fiber: a powerful single-cell model for cell wall and cellulose research. Front Plant Sci. 2012:3:104.

33. Guo $Y$, Pang $C$, Jia $X, M a ~ Q$, Dou L, Zhao F, Gu L, Wei H, Wang H, Fan S, et al. An NAM Domain Gene, GhNAC79, Improves Resistance to Drought Stress in Upland Cotton. Front Plant sci. 2017;8:1657

34. He X, Zhu L, Xu L, Guo W, Zhang X. GhATAF1, a NAC transcription factor, confers abiotic and biotic stress responses by regulating phytohormonal signaling networks. Plant Cell Rep. 2016:35(10):2167-79.

35. Meng C, Cai C, Zhang T, Guo W. Characterization of six novel NAC genes and their responses to abiotic stresses in Gossypium hirsutum L. Plant Sci. 2009;176(3):352-9. 
36. Huang G-Q, Li W, Zhou W, Zhang J-M, Li D-D, Gong S-Y, Li X-B. Seven cotton genes encoding putative NAC domain proteins are preferentially expressed in roots and in responses to abiotic stress during root development. Plant Growth Regul. 2013;71(2):101-12.

37. Shah ST, Pang C, Hussain A, Fan S, Song M, Zamir R, Yu S. Molecular cloning and functional analysis of NAC family genes associated with leaf senescence and stresses in Gossypium hirsutum L. Plant Cell Tiss Org. 2014;117(2):167-86.

38. Li F, Fan G, Wang K, Sun F, Yuan Y, Song G, Li Q, Ma Z, Lu C, Zou C, et al. Genome sequence of the cultivated cotton Gossypium arboreum. Nat Genet. 2014:46(6):567-72.

39. Liu X, Zhao B, Zheng HJ, Hu Y, Lu G, Yang CQ, Chen JD, Chen JJ, Chen DY, Zhang $L$, et al. Gossypium barbadense genome sequence provides insight into the evolution of extra-long staple fiber and specialized metabolites. Sci Rep. 2015;5:14139.

40. Wang K, Wang Z, Li F, Ye W, Wang J, Song G, Yue Z, Cong L, Shang H, Zhu $\mathrm{S}$, et al. The draft genome of a diploid cotton Gossypium raimondii. Nat Genet. 2012;44(10):1098-103.

41. Yuan D, Tang Z, Wang M, Gao W, Tu L, Jin X, Chen L, He Y, Zhang L, Zhu L, et al. The genome sequence of Sea-Island cotton (Gossypium barbadense) provides insights into the allopolyploidization and development of superior spinnable fibres. Sci Rep. 2015;5:17662.

42. Zhang T, Hu Y, Jiang W, Fang L, Guan X, Chen J, Zhang J, Saski CA, Scheffler BE, Stelly DM, et al. Sequencing of allotetraploid cotton (Gossypium hirsutum L. acc. TM-1) provides a resource for fiber improvement. Nat Biotechnol. 2015;33(5):531-7.

43. Shang H, Li W, Zou C, Yuan Y. Analyses of the NAC transcription factor gene family in Gossypium raimondii Ulbr: chromosomal location, structure, phylogeny, and expression patterns. J Plant Biol. 2013;55(7):663-76.

44. Shang H, Wang Z, Zou C, Zhang Z, Li W, Li J, Shi Y, Gong W, Chen T, Liu A, et al. Comprehensive analysis of NAC transcription factors in diploid Gossypium: sequence conservation and expression analysis uncover their roles during fiber development. Sci China Life Sci. 2016;59(2):142-53.

45. Riano-Pachon DM, Ruzicic S, Dreyer I, Mueller-Roeber B. PInTFDB: an integrative plant transcription factor database. BMC Bioinf. 2007:8:42.

46. Kim HJ, Nam HG, Lim PO. Regulatory network of NAC transcription factors in leaf senescence. Curr Opin Plant Biol. 2016;33:48-56.

47. Senchina DS, Alvarez I, Cronn RC, Liu B, Rong J, Noyes RD, Paterson AH, Wing RA, Wilkins TA, Wendel JF. Rate variation among nuclear genes and the age of polyploidy in Gossypium. Mol Biol Evol. 2003;20(4):633-43.

48. Wang K, Guo W, Yang Z, Hu Y, Zhang W, Zhou B, Stelly DM, Chen ZJ, Zhang T. Structure and size variations between $12 \mathrm{~A}$ and $12 \mathrm{D}$ homoeologous chromosomes based on high-resolution cytogenetic map in allotetraploid cotton. Chromosoma. 2010;119(3):255-66.

49. De Clercq I, Vermeirssen V, Van Aken O, Vandepoele K, Murcha MW, Law SR, Inze A, Ng S, Ivanova A, Rombaut D, et al. The membrane-bound NAC transcription factor ANAC013 functions in mitochondrial retrograde regulation of the oxidative stress response in Arabidopsis. Plant Cell. 2013; 25(9):3472-90.

50. Ng S, Ivanova A, Duncan O, Law SR, Van Aken O, De Clercq I, Wang Y, Carrie $C, X u L, K$ miec B, et al. A membrane-bound NAC transcription factor, ANAC017, mediates mitochondrial retrograde signaling in Arabidopsis. Plant Cell. 2013;25(9):3450-71.

51. Yogendra KN, Sarkar K, Kage U, Kushalappa AC. Potato NAC43 and MYB8 mediated transcriptional regulation of secondary Cell Wall biosynthesis to contain Phytophthora infestans infection. Plant Mol Biol Rep. 2017;35(5):519-33.

52. Ullah $A$, Sun $H$, Yang $X$, Zhang $X$. Drought coping strategies in cotton: increased crop per drop. Plant Biotechnol J. 2017;15(3):271-84.

53. Nakashima K, Takasaki H, Mizoi J, Shinozaki K, Yamaguchishinozaki K. NAC transcription factors in plant abiotic stress responses. BBA-Gene Regul Mech. 2012;1819(2):97-103

54. Gunapati S, Naresh R, Ranjan S, Nigam D, Hans A, Verma PC, Gadre R, Pathre UV, Sane AP, Sane VA. Expression of GhNAC2 from G-herbaceum, improves root growth and imparts tolerance to drought in transgenic cotton and Arabidopsis. Sci Rep. 2016;6:24978.

55. Fang Y, You J, Xie K, Xie W, Xiong L. Systematic sequence analysis and identification of tissue-specific or stress-responsive genes of NAC transcription factor family in rice. Mol Gen Genomics. 2008;80(6):547-63.

56. Jiang Y, Deyholos MK. Comprehensive transcriptional profiling of $\mathrm{NaCl}$ stressed Arabidopsis roots reveals novel classes of responsive genes. BMC Plant Biol. 2006;6:25.
57. Zhu T, Liang C, Meng Z, Sun G, Meng Z, Guo S, Zhang R. CottonFGD: an integrated functional genomics database for cotton. BMC Plant Biol. 2017;17:101.

58. Kumar S, Stecher G, Tamura K. MEGA7: molecular evolutionary genetics analysis version 7.0 for bigger datasets. Mol Biol Evol. 2016;33(7):1870-4.

59. Lescot M, Dehais $P$, Thijs $G$, Marchal $K$, Moreau $Y$, Van de Peer $Y$, Rouze $P$, Rombauts S. PlantCARE, a database of plant cis-acting regulatory elements and a portal to tools for in silico analysis of promoter sequences. Nucleic Acids Res. 2002;30(1):325-7.

60. Sun H, Chen L, Li J, Hu M, Ullah A, He X, Yang X, Zhang X. The JASMONATE ZIM-domain gene family mediates JA signaling and stress response in cotton. Plant Cell Physiol. 2017;58(12):2139-54.

61. Yang Z. PAML 4: phylogenetic analysis by maximum likelihood. Mol Biol Evol. 2007;24(8):1586-91

62. Wang $M$, Tu L, Lin M, Lin Z, Wang P, Yang Q, Ye Z, Shen C, Li J, Zhang L, et al. Asymmetric subgenome selection and cis-regulatory divergence during cotton domestication. Nat Genet. 2017;49(4):579-87.

63. Sturn A, Quackenbush J, Trajanoski Z. Genesis: cluster analysis of microarray data. Bioinformatics. 2002;18(1):207-8.

64. Langfelder P, Horvath S. WGCNA: an R package for weighted correlation network analysis. BMC Bioinf. 2008;9:559.

65. Xie C, Mao X, Huang J, Ding Y, Wu J, Dong S, Kong L, Gao G, Li CY, Wei L. KOBAS 2.0: a web server for annotation and identification of enriched pathways and diseases. Nucleic Acids Res. 2011;39(Web Server issue):W316-22.

66. Hu H, He X, Tu L, Zhu L, Zhu S, Ge Z, Zhang X. GhJAZ2 negatively regulates cotton fiber initiation by interacting with the R2R3-MYB transcription factor GhMYB25-like. Plant J. 2016;88(6):921-35.

67. Yoo SD, Cho YH, Sheen J. Arabidopsis mesophyll protoplasts: a versatile cell system for transient gene expression analysis. Nat Protoc. 2007;2(7):1565-72.

\section{Ready to submit your research? Choose BMC and benefit from:}

- fast, convenient online submission

- thorough peer review by experienced researchers in your field

- rapid publication on acceptance

- support for research data, including large and complex data types

- gold Open Access which fosters wider collaboration and increased citations

- maximum visibility for your research: over $100 \mathrm{M}$ website views per year

At BMC, research is always in progress.

Learn more biomedcentral.com/submissions 